\title{
An Improved General Type-2 Fuzzy Approach for Planning Energy Resources Systems with Air Quality Requirements
}

\author{
Y. Cheng ${ }^{1}$, L. Jin ${ }^{1}$, D. Lin ${ }^{1}$, Y. Pan ${ }^{1}$ and X. Chen ${ }^{1}$ \\ ${ }^{1}$ College of Environmental Science and Engineering, Xiamen University of Technology, Xiamen 361024, China
}

Received 27 March 2021; revised 25 April 2021; accepted 29 April 2021; published online 16 September 2021

\begin{abstract}
Due to economic development and the improvement of people's living standards, questions that the imbalance of energy supply and demand as well as the environmental damage have become increasingly prominent. To study the contradiction between energy and environment, and considering there are a lot of uncertain factors in the actual planning, this paper improves a chance-constrained interval type- 2 fuzzy method used in linear programming and extends the interval type-2 fuzzy number to the general type- 2 fuzzy number by introducing the secondary membership function that makes up for the loss of uncertainty due to the fixed value of the second membership function and optimizes the accuracy of the probability in the original method. By applying the new method to the energy planning system model, an optimal planning plan with the goal of reducing air pollutant emissions as well as the lowest overall operating cost can be obtained. Compared with the interval fuzzy method, the new method proposed in this paper is able to deal with the uncertainty in the system better, and provides more accurate solutions under different satisfaction levels.
\end{abstract}

Keywords: type-2 fuzzy number, uncertainty, energy programming, air pollution emissions

\section{Introduction}

Energy is essential to ensure human survival, social development and civilization inheritance. To meet human needs, the energy demand and utilization is increasing rapidly, but major energy sources such as coal, oil, and natural gas cannot be developed and utilized indefinitely. To achieve sustainable development, it is necessary to reduce the dependence on fossil fuels and should be replaced with clean energy gradually. By 2020, China's population will reach 1.40 billion which is in stark contrast to that of 1.34 billion in 2010 (China Statistical Yearbook, 2020). Accordingly, energy demand continues to increase, and environmental problems caused by energy will become much more serious, meaning China is not only facing population pressure (population growth exceeds economic growth), energy and environmental issues are also imminent (BP in China, 2015).

Energy system and environmental system are not two independent systems, they will influence and interact with each other. The energy production and conversion process will cause corresponding environmental problems (Guo et al., 2019). Relevant data show that $75 \%$ of global carbon dioxide emissions are the main source of pollution from the combustion of coal and oil. Coal combustion produces $70 \sim 80 \%$ of sulfur dioxide, nitrogen oxides, PM 2.5 and PM 10 and the impact on the envi-

${ }^{*}$ Corresponding author. Tel.: 086-157-5077-7872.

E-mail address: jinlei777@ outlook.com (L.Jin).

ISSN: 2663-6859 print/2663-6867 online

(c) 2021 ISEIS All rights reserved. doi:10.3808/jeil.202100071 ronmental system will be negatively fed back to the energy system. For example, polluted water resources will reduce the use of water resources; greenhouse gases emitted into the atmosphere will cause the greenhouse effect, leading to the melting of two-level glaciers; reducing freshwater resources, and some temporarily unexploitable natural gas resources will leak ahead of time. Therefore, research on energy must not only consider energy issues, but also take the impact of energy on the environment into account (Zeng et al., 2020).

In addition, the district energy system is a large and complex energy system composed of multiple subsystems and the entire process from energy extraction to energy consumption is included in the energy system (Lu et al., 2020). There are also various factors affecting energy activities in the regional energy system, including domestic and foreign politics as well as economics, and regional environment. Since multiple factors play a role in the energy system at the same time, the energy system has multiple uncertainties. Energy prices, utilization rates, conversion technologies, supply, demand and energy will also change with the alteration of time and space and the change is dynamic. The energy system and the environmental system influence each other. On the one hand, energy activities emit gas into the atmosphere, which will affect air quality through physical, chemical and biological effects. On the other hand, changes in air quality will adversely affect the process of energy extraction, conversion and utilization (Wu et al., 2021).

At present, there are lots of research on energy planning optimization. With the goal of maximizing the system revenue and energy utilization of the micro-energy grid, Chen et al. 
(2019) provides decision-makers with a variety of optimal scheduling schemes. Simultaneously, with the goal of minimizing the total cost of the system, carbon dioxide emissions, and the lack of energy supply, the two operating strategies were considered to optimize the configuration of the system ( $\mathrm{Li}$ et al., 2018a). Liu et al. (2019) comprehensively considered the economic impact and environmental benefits and transformed the multi-objective optimization problem into a single objective problem in a weighted manner. Kiptoo et al. (2019) took the short-term variability into account in the long-term planning and reduced the total cost of the system with the goal of minimizing the energy loss rate. The above studies comprehensively considered economic, energy consumption, environmental protection and other indicators in the optimization research, yet did not consider the uncertainty in the system. In fact, different production processes and energy conversion methods in the energy system are affected by a variety of uncertain factors and ignoring the uncertainties will reduce the accuracy of planning (Li et al., 2018b).

In view of the uncertainty in the planning system, scholars have conducted optimization studies in three aspects: probability, fuzziness, and interval. Emeç and Akkaya (2021) developed a fuzzy optimal renewable energy model (F-OREM) to solve energy problems involving fuzzy parameters; Akram et al. (2021) extended the concept of crisp linear programming problem in Pythagorean fuzzy environment based on triangular Pythagorean fuzzy numbers; Mohan et al. (2021) used dual simplex algorithm and sensitivity analysis to solve intuitionistic fuzzy linear programming problems; Milanovic and Komatina (2020) used Fuzzy AHP and fuzzy linear programming on the determination of the optimal production plan. Alizdeh and Saeidi (2020) defined the duration of activities and the amount of resources used by each activity as a fuzzy membership function when considering project scheduling considering risks and resource constraints in an uncertain environment.

Since Zadeh proposed fuzzy set theory (Zadeh, 1965), fuzzy theory and type-two fuzzy set theory have developed rapidly. Type-2 fuzzy set theory introduces a secondary membership function on the traditional fuzzy theory, and fuzzifies the membership of each point into a new membership function, which greatly enhances the performance of fuzzy theory in dealing with uncertainty and is widely used in the modeling and control of complex industrial processes. In the linear programming problem, there are often a mass of uncertainties in the various coefficients in the planning system, incorporating the uncertainty of human observation and concepts. It is very suitable to use the type-two fuzzy set theory to cope with these double uncertainties.

Scholars have done a lot of research on various properties of fuzzy sets, including the operations between fuzzy sets, the centroid and similarity of fuzzy sets (Chi and Vincent, 2018; Chen, 2019; Naimi, 2020), and the entire fuzzy logic system has fully developed (Zhao, 2019). The preliminary data input fuzzy rules include Mamdani fuzzy rules and TSK fuzzy rules (Mamdani, 1974; Takagi and Sugeno, 1985); however, there are few researches on the ranking of fuzzy numbers. At present, there are direct ranking methods using centroids for interval type-2 fuzzy sets (Wu and Mendel, 2009), and Figueroa et al. (2018) proposed a ranking method based on Yager index and Srinivasan proposed a ranking method based on probability degree (Srinivasan and Geetharamani, 2016).

The sorting method proposed by Srinivasan and Geetharamani has some shortcomings. First, it obtains the value of the maximum degree of membership by calculating the intersection of the upper boundary and the lower boundary of the membership functions of two fuzzy numbers. However, the value of the degree of membership corresponding to the intersection of the upper membership function is always greater than the value of the degree of membership corresponding to the intersection of the lower membership function, which makes it essentially a sorting method for calculating type-1 fuzzy numbers. On the other hand, this method uses perfect interval type-2 fuzzy numbers. These fuzzy numbers set the value of the secondary membership function to 1 , which causes the uncertainty contained in the secondary membership function in the fuzzy number to be lost. This also leads to the weaker performance of the modified method in dealing with the uncertainty in linear programming (Mendel, 2017).

This paper, based on the numerical calculation ranking method proposed by Srinivasan and Geetharamani, takes the secondary membership function into account and uses the centroid of the secondary membership function to find the optimal solution under the specified probability, proposing an improved sorting calculation method. The improved method obtains the maximum probability value by calculating the intersection of the centroid trajectory curves of the secondary membership functions of the two fuzzy numbers. By setting different degrees of possibility according to requirements, fuzzy relations can be transformed into precise inequalities. The structure of this paper is as follows: In the second section, we introduce some basic knowledge of type-2 fuzzy set theory and fuzzy numbers. In the third section, a simulated regional energy system model is established, and the improved fuzzy linear programming method is used to solve the optimal solution of the system under the specified air pollutant emissions. The fourth and fifth section summarizes the advantages and disadvantages of this method and put forward some directions that can be further studied.

\section{Methodology}

\subsection{Perfectly Interval Type-2 Fuzzy Number}

Fuzzy set theory will not be repeated in this article. For details, please refer to Professor Zardeh's paper. The interval trapezoidal fuzzy set is an important part of the concept in type2 fuzzy sets, while the membership function of the perfectly normal interval type-2 fuzzy number (PnIT2TrFN) adopts the form of its interval trapezoid shape. The definition of PnIT2TrFN is as follows (Srinivasan and Geetharamani, 2016): let $\tilde{A}$ be an interval type-2 fuzzy number (Figure 1) defined on the universe of discourse $X$, then its lower membership function and upper membership function can be expressed as $\tilde{A}^{L}=\left(a_{2}^{L}\right.$, $\left.a_{3}^{L}, \alpha_{L}, \beta_{L}\right)$ and $\tilde{A}^{U}=\left(a_{2}^{U}, a_{3}^{U}, \alpha_{U}, \beta_{U}\right)$, respectively. $\left[a_{2}^{L}, a_{3}^{L}\right]$ is the core 
of $\tilde{A}^{L}$ and $\tilde{A}^{U}$, respectively $\alpha_{L}, \beta_{L}>0$ is the left-hand spreads and the $\alpha_{U}, \beta_{U}>0$ is the right-hand spreads. The low and upper membership function are both "normal" and "convex". The membership functions of them are expressed as follows:

$\underline{u}_{\tilde{A}}(x)=\left\{\begin{array}{cc}\frac{\left(x-a_{2}^{L}+\alpha_{L}\right)}{\alpha_{L}}, & a_{2}^{L}-\alpha_{L} \leq x \leq a_{2}^{L} \\ 1, & a_{2}^{L} \leq x \leq a_{3}^{L} \\ -\frac{\left(x-a_{3}^{L}-\beta_{L}\right)}{\beta_{L}}, & a_{3}^{L} \leq x \leq a_{3}^{L}+\beta_{L} \\ 0, & \text { otherwise }\end{array}\right.$

$\bar{u}_{\tilde{A}}(x)=\left\{\begin{array}{cc}\frac{\left(x-a_{2}^{U}+\alpha_{U}\right)}{\alpha_{U}}, & a_{2}^{U}-\alpha_{U} \leq x \leq a_{2}^{U} \\ 1, & a_{2}^{U} \leq x \leq a_{3}^{U} \\ -\frac{\left(x-a_{3}^{U}-\beta_{U}\right)}{\beta_{U}}, & a_{3}^{U} \leq x \leq a_{3}^{U}+\beta_{U} \\ 0, & \text { otherwise }\end{array}\right.$

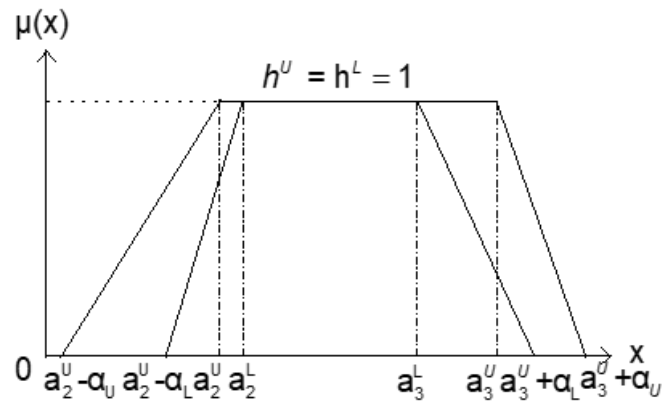

Figure 1. Perfectly normal interval type-2 trapezoidal fuzzy number (Srinivasan and Geetharamani, 2016).

\subsection{Arithmetic Operations}

Let $\quad \tilde{A}=\left(a_{2}^{L}, a_{3}^{L}, \alpha_{L}, \beta_{L}\right),\left(a_{2}^{U}, a_{3}^{U}, \alpha_{U}, \beta_{U}\right) \quad$ and $\tilde{B}=\left(b_{2}^{L}, b_{3}^{L}, \gamma_{L}, \theta_{L}\right),\left(b_{2}^{U}, b_{3}^{U}, \gamma_{U}, \theta_{U}\right)$ be two perfectly interval fuzzy number, then $\tilde{A}+\tilde{B}=\left(a_{2}^{L}+b_{2}^{L}, a_{3}^{L}+b_{3}^{L}, \alpha_{L}+\gamma_{L}, \beta_{L}+\theta_{L}\right),\left(a_{2}^{U}+b_{2}^{U}, a_{3}^{U}+\right.$ $\left.b_{3}^{U}, \alpha_{U}+\gamma_{U}, \beta_{U}+\theta_{U}\right) ;$ similarly, $\tilde{A}-\tilde{B}=\left(a_{2}^{L}-b_{2}^{L}, a_{3}^{L}-b_{3}^{L}, \alpha_{L}+\gamma_{L}\right.$, $\left.\beta_{L}+\theta_{L}\right),\left(a_{2}^{U}-b_{2}^{U}, a_{3}^{U}-b_{3}^{U}, \alpha_{U}+\gamma_{U}, \beta_{U}+\theta_{U}\right)$. For $\mu \in R$ :

$\mu \tilde{A}=\left\{\begin{array}{cc}\left(\left(\mu a_{2}^{L}, \mu a_{3}^{L}, \mu \alpha_{L}, \mu \beta_{L}\right),\left(\mu a_{2}^{U}, \mu a_{3}^{U}, \mu \alpha_{U}, \mu \beta_{U}\right)\right) ; & \mu>0 \\ \left(\left(\mu a_{3}^{L}, \mu a_{2}^{L},|\mu| \beta_{U},|\mu| \alpha_{U}\right),\left(\mu a_{3}^{U}, \mu a_{2}^{U},|\mu| \beta_{L},|\mu| \alpha_{L}\right)\right) ; & \mu<0\end{array}\right.$

\subsection{Improved Ranking Method}

\subsubsection{Probability Degree Method}

In Srinivasan and Geetharamani's study, a probability degree method (Figures $2 \sim 4$ ) is proposed to explain the ambiguity of the left and right coefficients in the constraint condition and decompose it into two simple linear equation with crisp number (Srinivasan and Geetharamani, 2016). A conventional linear program is as follows:

$$
\begin{aligned}
& \text { Max/Minf } f=C X ; \\
& \text { s.t. } \quad A X \leq B ; \\
& \quad X \geq 0
\end{aligned}
$$

Then introduce fuzzy numbers on the left- and right-hand sides of the constraints, the original formula is converted to:

$$
\begin{aligned}
& \text { Max/Min } f=C X ; \\
& \text { s.t. } \quad \tilde{A} X \leq \tilde{B} ; \\
& X \geq 0 ; \\
& \quad \operatorname{Pos}[\tilde{A} \leq \tilde{B}]
\end{aligned}
$$

By introducing the RITT2FLP-CPDs model of Jin et al. (2019), the conceptual processing model of possibility is used. The formula can be transformed into:

$$
\begin{aligned}
& \operatorname{Max} / \operatorname{Minf}=\sum_{j=1}^{n} \otimes_{m}\left(c_{j}\right) \otimes_{m}\left(x_{j}\right) \\
& \text { s.t. } \bigotimes_{m}\left(\tilde{a}_{i 1}^{L}\right) \bigotimes_{m}\left(x_{1}\right)+\bigotimes_{m}\left(\tilde{a}_{i 2}^{L}\right) \bigotimes_{m}\left(x_{2}\right)+\ldots+ \\
& \bigotimes_{m}\left(\tilde{a}_{i n}^{L}\right) \bigotimes_{m}\left(x_{n}\right) \leq \bigotimes_{m}\left({ }^{P o s} \tilde{b}_{i}^{L}\right), i=1,2, \ldots, m \\
& \otimes_{m}\left(x_{j}\right) \geq 0, j=1, \ldots, N
\end{aligned}
$$

$$
\begin{aligned}
& \text { Max } / \operatorname{Minf}=\sum_{j=1}^{n} \bigotimes_{m}\left(c_{j}\right) \bigotimes_{m}\left(x_{j}\right) \\
& \begin{array}{l}
\text { s.t. } \otimes_{m}\left(\tilde{a}_{i 1}^{U}\right) \bigotimes_{m}\left(x_{1}\right)+\bigotimes_{m}\left(\tilde{a}_{i 2}^{U}\right) \bigotimes_{m}\left(x_{2}\right)+\ldots+ \\
\bigotimes_{m}\left(\tilde{a}_{i n}^{U}\right) \bigotimes_{m}\left(x_{n}\right) \leq \bigotimes_{m}\left({ }^{P o s} \bar{b}_{i}^{L}\right), i=1,2, \ldots, m \\
\quad \bigotimes_{m}\left(x_{j}\right) \geq 0, j=1, \ldots, N
\end{array}
\end{aligned}
$$

which is equivalent to:

$$
\begin{array}{ll}
\text { Max/Min } & f=\sum_{j=1}^{n} c_{j} x_{j} \\
\text { s.t. } \quad & \sum_{j=1}^{n} \tilde{a}_{i j}^{L} \leq \tilde{b}_{i}, i=1,2, \ldots, m \\
& x_{j} \geq 0, \quad j=1,2, \ldots, n
\end{array}
$$

$\operatorname{Max} / \operatorname{Min} f=\sum_{j=1}^{n} c_{j} x_{j}$

$$
\begin{array}{ll}
\text { s.t. } \quad & \sum_{j=1}^{n} \tilde{a}_{i j}^{U} \leq \tilde{b}_{i}, i=1,2, \ldots, m \\
& x_{j} \geq 0, \quad j=1,2, \ldots, n
\end{array}
$$

To deal with the fuzzy coefficient on the left-hand side of the constraint function, we introduce the concept of possibility degree to explain it. Let $\tilde{A}=\left(\left(a_{2}^{L}, a_{3}^{L}, \alpha_{L}, \beta_{L}\right),\left(a_{2}^{U}, a_{3}^{U}, \alpha_{U}, \beta_{U}\right)\right)$ be a PnIT2TrFN, define the $\alpha$-cut of PnIT2TrFN $\tilde{A}$ as ${ }^{\alpha} \tilde{A}=$ $\left\{(x, u) \mid J_{x} \geq \alpha, u \in[0,1]\right\}$, which is bounded by two intervals:

$\begin{aligned}{ }^{\alpha} \underline{\mu}_{\tilde{A}} & =\left\{\left(x, \underline{\mu}_{\tilde{A}}(x)\right) \mid \underline{\mu}_{\tilde{A}}(x) \geq \alpha, \forall \alpha \in[0,1]\right\} \\ { }^{\alpha} \bar{\mu}_{\tilde{A}} & =\left\{\left(x, \bar{\mu}_{\tilde{A}}(x)\right) \mid \bar{\mu}_{\tilde{A}}(x) \geq \alpha, \forall \alpha \in[0,1]\right\}\end{aligned}$ 
According to the Figure 1, let $\tilde{A}=\left(\left(a_{2}^{L}, a_{3}^{L}, \alpha_{L}, \beta_{L}\right),\left(a_{2}^{U}, a_{3}^{U}\right.\right.$, $\left.\left.\alpha_{U}, \beta_{U}\right)\right)$ and $\tilde{B}=\left(\left(b_{2}^{L}, b_{3}^{L}, \gamma_{L}, \theta_{L}\right),\left(b_{2}^{U}, b_{3}^{U}, \gamma_{U}, \theta_{U}\right)\right)$ be two perfectly interval type-2 fuzzy numbers, make Pos $\left[\tilde{A}^{L} \leq \tilde{B}^{L}\right] \geq p, p \in(0,1]$ and $\operatorname{Pos}\left[\tilde{A}^{U} \leq \tilde{B}^{U}\right] \geq p$, we get:

$b_{3}^{L}-a_{2}^{L} \geq(p-1)\left(\theta_{L}+\alpha_{L}\right)$

$b_{3}^{U}-a_{2}^{U} \geq(p-1)\left(\theta_{U}+\alpha_{U}\right)$

Then Equations (6) and (7) can be converted to:

$\operatorname{Max} / \operatorname{Min} f=\sum_{j=1}^{n} c_{j} x_{j}$

s.t. $\quad \operatorname{Pos}\left(\tilde{a}_{i 1}^{L} x_{1}+\tilde{a}_{i 2}^{L} x_{1}+\ldots+\tilde{a}_{i n}^{L} x_{1} \leq \tilde{b}_{i}^{L}\right) \geq p, \quad i=1,2, \ldots, m$

$x_{j} \geq 0, j=1,2, \ldots, n$

$\operatorname{Max} / \operatorname{Min} f=\sum_{j=1}^{n} c_{j} x_{j}$

s.t. $\quad \operatorname{Pos}\left(\tilde{a}_{i 1}^{U} x_{1}+\tilde{a}_{i 2}^{U} x_{1}+\ldots+\tilde{a}_{i n}^{U} x_{1} \leq \tilde{b}_{i}^{U}\right) \geq p, i=1,2, \ldots, m$

$x_{j} \geq 0, j=1,2, \ldots, n$

From Equations (9) and (10), the optimal solution is as follows:

$$
\begin{array}{ll}
\underline{F}= & \operatorname{Max} / \operatorname{Min} f=\sum_{j=1}^{n} c_{j} x_{j} \\
\text { s.t. } & \underline{b}^{U}-\underline{A}^{L} x \geq(p-1)(\underline{\alpha} x+\underline{\theta}), i=1,2, \ldots, m \\
& x_{j} \geq 0, \\
& j=1,2, \ldots, n
\end{array}
$$

$\bar{F}=\operatorname{Max} / \operatorname{Min} f=\sum_{j=1}^{n} c_{j} x_{j}$

s.t. $\quad \bar{b}^{U}-\bar{A}^{L} x \geq(p-1)(\bar{\alpha} x+\bar{\theta}), i=1,2, \ldots, m$

$x_{j} \geq 0$,

$j=1,2, \ldots, n$

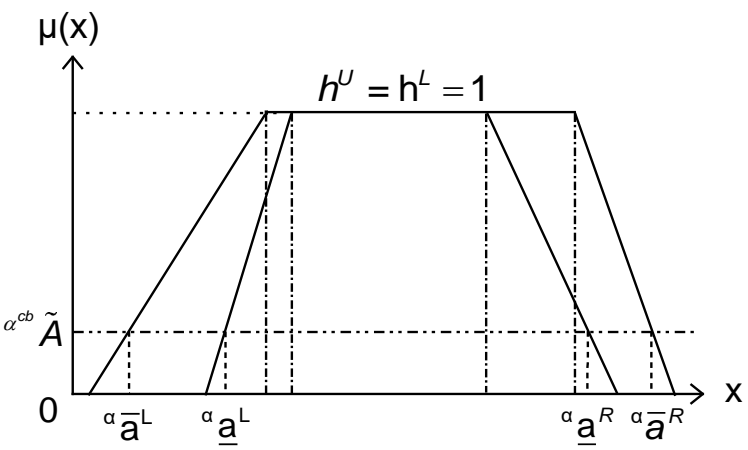

Figure 2. PnIT2TrFN under probability degree $\alpha$ (Srinivasan and Geetharamani, 2016).

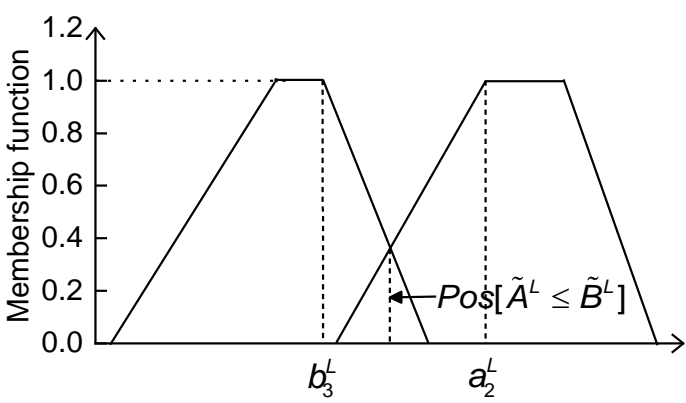

Figure 3. $\operatorname{Pos}\left[\tilde{A}^{L} \leq \tilde{B}^{L}\right]<1$, because $\tilde{A}^{L}>\tilde{B}^{L}$.

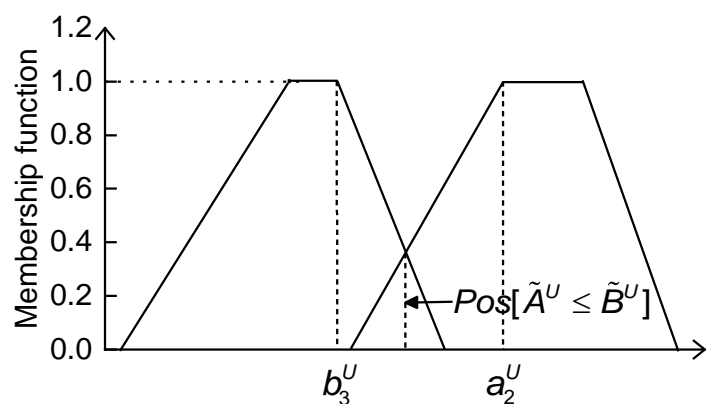

Figure 4. $\operatorname{Pos}\left[\tilde{A}^{U} \leq \tilde{B}^{U}\right]<1$, because $\tilde{A}^{U}>\tilde{B}^{U}$.

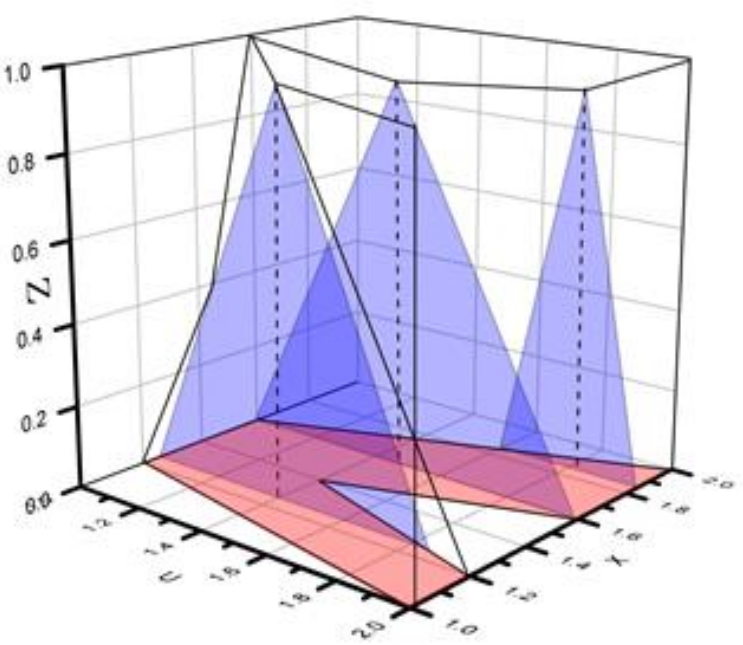

Figure 5. General fuzzy number form.

\subsubsection{Extended to General Type-2 Fuzzy Numbers}

The above method omits the information of the secondary membership function of the type-2 fuzzy number in the calculation process. This study replaces the interval fuzzy number in the above method with a general type fuzzy number, discretizes the $\mathrm{X}$-axis and assigns each point a secondary membership function, thereby enhancing its ability to deal with uncertainty in linear programming systems (Figure 5).

Using the weighted sum method to find the centroid of the secondary membership function of each point. Let $X_{0}$ be a point in a perfectly general fuzzy number $\tilde{A}=\left(\left(a_{2}^{L}, a_{3}^{L}, \alpha_{L}, \beta_{L}\right),\left(a_{2}^{U}, a_{3}^{U}\right.\right.$, 
$\left.\left.\alpha_{U}, \beta_{U}\right)\right)$, secondary membership function $\mu_{x_{i}}=\left\{x_{i}, \mu_{A_{i}}\left(x_{i}\right) \mid x_{i} \in(0\right.$, $1]\}$, then the centroid of the secondary membership function can be expressed as:

$c\left(\mu_{x_{i}}\right)=\frac{x_{i} \mu_{A_{i}}\left(x_{i}\right)}{\mu_{A_{i}}\left(x_{i}\right)}, x_{i} \in(0,1]$

By calculating the centroid of the secondary membership function of each discrete point, the centroid trajectory in the first-level membership function interval could be obtained (as shown by the blue line in Figure 6). The higher the degree of discretization, the more accurate the trajectory obtained, and the corresponding calculation amount is greater. In this study, the interval where the trajectory intersection point is first calculated, which reduces the amount of calculation. The interval is shown as $\left[c^{U}, c^{L}\right]$, that $\tilde{A}=\left(\left(a_{2}^{L}, a_{3}^{L}, \alpha_{L}, \beta_{L}\right),\left(a_{2}^{U}, a_{3}^{U}, \alpha_{U}, \beta_{U}\right)\right), \tilde{B}=$ $\left(\left(b_{2}^{L}, b_{3}^{L}, \gamma_{L}, \theta_{L}\right),\left(b_{2}^{U}, b_{3}^{U}, \gamma_{U}, \theta_{U}\right)\right)$, the following formulas can be obtained:

$$
\begin{aligned}
& c^{U}=\frac{b_{3}^{U}-a_{2}^{L}+\theta_{U}+\alpha_{L}}{\theta_{U}+\alpha_{L}} \\
& c^{L}=\frac{b_{3}^{L}-a_{2}^{U}+\theta_{L}+\alpha_{U}}{\theta_{L}+\alpha_{U}}
\end{aligned}
$$

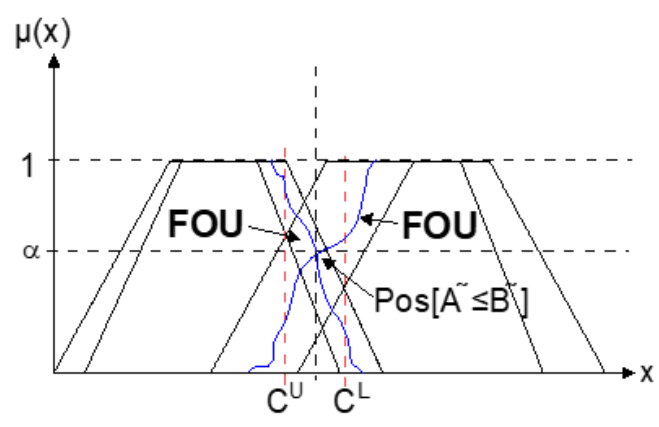

Figure 6. The centroid locus of the secondary membership function.

Based on the centroid of the secondary membership function in the above interval, we can use matlab fitting function to obtain the centroid trajectory curve. By calculating the intersection point of the centroid trajectory of the two secondary membership functions, the variable value $X_{\alpha}$ corresponding to $\alpha$-cut can be obtained. Thus, the linear programming model (5) can be converted to:

$$
\begin{array}{ll}
\text { Max / Min } & f=C X ; \\
\text { s.t. } & \\
& \\
& \operatorname{FOU}_{A}\left(X_{\alpha}\right) \geq \alpha, \\
& X_{\alpha} \in\left(c^{U}, c^{L}\right)
\end{array}
$$

when fitted curve of the centroid trajectory is approximately regarded as a straight line, the model can be transformed into:

$$
\begin{array}{ll}
\text { Min } & f=\sum_{j=1}^{n} c_{j} x_{j} \\
\text { s.t. } & b^{F O U}-A^{F O U} x \geq(p-1)\left(\alpha^{F O U} x+\theta^{F O U}\right) \\
& x_{j} \geq 0
\end{array}
$$

\section{Application of the Developed Method to Energy Programming}

\subsection{Statement of Research Issues}

Energy and environment are closely linked, whether in the process of energy extraction, transportation, processing, conversion, and consumption, energy activities will put pressure on the environment, and the environment will in turn affect energy activities. With the rapid economic growth, the demand for energy continues to increase. The pressure of energy activities on the environment is becoming more and more obvious, causing many environmental problems. The main products of energy activities emitted to the atmosphere are sulfur dioxide and nitrogen oxides. The main source of sulfur dioxide is sulfur-containing coal, mainly produced in the process of thermal power generation and energy consumption in the terminal sector. Nitrogen oxides are mainly emitted by power plants, industrial sectors, and transportation sectors.

A simulated medium-sized district energy system is established in this paper. The energy types in the system are divided into primary energy and secondary energy. Primary energy includes coal, natural gas, hydropower, wind energy, nuclear energy, crude oil, etc., which can be directly used after mining. The secondary energy sources are energy sources such as diesel, gasoline, and electricity, which need to be processed before they can be used. The five main sectors of industry, agriculture, transportation, commerce and residents serve as energy demand terminals. In the process of energy extraction, processing, transformation and consumption, energy activities will have an impact on the environment, polluting gases such as sulfur dioxide and nitrogen oxides into the atmosphere.

The energy supply in the system is divided into local production and external transfer. Normally, the price of external transfer will be higher than the price of local production. Different energy requirements, output efficiencies and pollutant emissions are also different. How to adjust the energy production structure to minimize the overall system operating cost while meeting the pollution gas emission standards is the main problem to be studied in this article. In the model of this article, during the calculation of the centroid trajectory of the fuzzy number, it is secondary membership function is idealized as a normal triangular membership function.

\subsection{Model Formulation}

The regional energy system studied is composed of the following four parts: energy supply, energy conversion/processing, energy/electricity demand, and environmental emissions. The types of energy considered are coal, diesel, gasoline, electricity, crude oil, natural gas. Electricity production mainly includes coal-fired power generation, natural gas power genera- 
tion, hydropower generation, wind power generation, and nuclear power generation. Pollutant emissions are mainly sulfur dioxide and nitrogen oxides.

The costs related to the objective function are the total cost of energy supply and production, the total cost of energy conversion, and the total cost of emission reduction (removal of pollutants). The total cost of energy supply mainly refers to the cost of local energy production and energy transfer from other area. The total cost of energy conversion is the operating cost of converting energy into electric energy. The total cost of emission reduction mainly refers to the cost of removing harmful gases generated by energy activities and energy-related activities. There is also a part of government subsidies for the subsidy cost of pollutants for power plants.

In this model, it is planned to study the energy planning plan within ten years, five years as a cycle. The goal of the energy system optimization model is to minimize the total cost of the system. The objective function of the district energy system is expressed as follows:

$$
\begin{aligned}
& \operatorname{Min} f^{ \pm}=\sum_{t=1}^{2} \sum_{j=1}^{6} I A_{j, t} \times I P_{j, t}+\sum_{t=1}^{2} \sum_{j=1}^{4} N A_{j, t} \times N P_{j, t} \\
& +\sum_{t=1}^{2} \sum_{k=1}^{5} E C_{k, t} \times X_{k, t}+\sum_{t=1}^{2} \sum_{i=1}^{2} X_{i, t} \times\left(C T_{i, t}-C P_{i, t}\right)
\end{aligned}
$$

where the $I A_{j, t}$ represents the total amount of energy transferred from outside, $I P_{j, t}$ the price of energy transferred from other places by different energy types (Table 1 ), $N A_{j, t}$ represents the total amount of energy produced locally, $N P_{j, t}$ represents the price of locally produced energy (Table 2), $E C_{k, t}$ represents the conversion cost of different energy sources (Table $3), X_{k, t}$ represents the total conversion of different energy sources, $C T_{i, t}$ means the unit cost of removing pollutants, and $C P_{i, t}$ means government environmental protection subsidy.

Table 1. Import Price

\begin{tabular}{lll}
\hline IP $\left(10^{6} \mathrm{CNY} / \mathrm{PJ}\right)$ & $t=1$ & $t=2$ \\
\hline Coal & $(33,35,3,5)$ & $(35,40,3,4)$ \\
Diesel & $(164,166,15,15)$ & $(166,170,16,16)$ \\
Gasoline & $(173,174,17,17)$ & $(175,180,17,18)$ \\
Electric power & $(107,114,10,11)$ & $(114,120,11,12)$ \\
Crude oil & $(139,141,12,13)$ & $(141,146,14,14)$ \\
Natural gas & $(82,89,8,9)$ & $(90,100,9,10)$ \\
\hline
\end{tabular}

Table 2. Local Production Price

\begin{tabular}{lll}
\hline NP $\left(10^{6} \mathrm{CNY} / \mathrm{PJ}\right)$ & $t=1$ & $t=2$ \\
\hline Coal & $(21,25,2,2)$ & $(25,27,2.5,2.7)$ \\
Diesel & $(116,123,11,12)$ & $(124,130,12,13)$ \\
Gasoline & $(120,130,12,12)$ & $(130,140,10,11)$ \\
Electric power & $(107,112,10,10)$ & $(112,120,11,10)$ \\
\hline
\end{tabular}

The model constraints mainly consider three aspects: energy supply and demand balance constraints, power supply and demand balance constraints, and pollutant emission constraints.
Specific constraints are as follows:

(1) Coal supply and demand balance constraints. In the energy system, coal mainly meets the needs of the power conversion industry and the coal needs of end customers (Table 4). There are two main methods for coal production: local production and transfer from other provinces:

$$
\begin{aligned}
& I A_{1, t}+N A_{1, t}-X_{1, t} \times C F E_{1, t} \geq D_{1, t} \\
& N A_{1, t} \leq T O K_{1, t}
\end{aligned}
$$

where the $C F E_{1, t}$ represents the efficiency of converting different types of energy into electrical energy (Table 5), the TOK ${ }_{1, t}$ represents the local production limit of different energy sources.

Table 3. Energy Conversion Cost

\begin{tabular}{lll}
\hline EC $\left(10^{6} \mathrm{CNY} / \mathrm{PJ}\right)$ & $t=1$ & $t=2$ \\
\hline Coal-fired & $(9.8,10.9,0.9,1)$ & $(9,10,0.8,1)$ \\
Natural gas & $(12.4,13,1,1)$ & $(12,12.5,1,1)$ \\
Hydraulic & $(60,69,5,6)$ & $(57,65,6,5)$ \\
Wind & $(109,120,11,12)$ & $(105,110,11,10)$ \\
Nuclear & $(12,13,1,1)$ & $(11,12,1,1.2)$ \\
\hline
\end{tabular}

Table 4. Terminal Demand in Different Periods

\begin{tabular}{lll}
\hline $\mathrm{D}(\mathrm{PJ})$ & $t=1$ & $t=2$ \\
\hline Coal & $(552,669,223,322)$ & $(600,700,60,70)$ \\
Diesel & $(148,154,14,15)$ & $(154,160,15,16)$ \\
Gasoline & $(107,118,10,11)$ & $(120,130,12,12)$ \\
Electric power & $(537,603,53,55)$ & $(600,700,60,65)$ \\
Crude oil & $(278,319,27,31)$ & $(300,350,30,35)$ \\
Natural gas & $(119,120,12,12)$ & $(120,130,11,11)$ \\
\hline
\end{tabular}

(2) Diesel supply and demand balance constraints. Diesel includes two types: locally produced and purchased from other provinces. Local production is mainly provided by refineries. The supply of diesel should be greater than or equal to the demand of the terminal sector:

$I A_{2, t}+N A_{2, t} \geq D_{2, t}$

$N A_{2, t} \leq \mathrm{TOK}_{2, t}$

(3) Gasoline supply and demand balance. There are two main sources of gasoline, like diesel: local refineries produced, and imported from other provinces:

$I A_{3, t}+N A_{3, t} \geq D_{3, t}$

$N A_{3, t} \leq \mathrm{TOK}_{3, t}$ 
(4) Crude oil supply and demand balance constraints. To meet the production of the refinery, the amount of crude oil transferred must be greater than or equal to the crude oil demand of the refinery:

$I A_{5, t} \geq D_{5, t}$

(5) Constraints on the balance of natural gas supply and demand. Natural gas demand mainly consists of natural gas power generation and terminal demand:

$I A_{6, t}-X_{2, t} \times C F E_{2, t} \geq D_{6, t}$

(6) Electricity supply and demand balance constraints. There are two main sources of electricity supply, one is local power generation and the amount transferred from other provinces:

$\sum_{i=1}^{5} X_{i, t}+I A_{4, t} \geq D_{4, t}$

$X_{i, t} \geq \operatorname{TON}_{i, t}$

where the $T O N_{i, t}$ represents the minimum production limit for each energy conversion method.

(7) Pollutant discharge constraints. In the process of using and using energy, pollutants are generated, in this model, pollutant emissions from the power industry and end users are mainly considered. The discharge of pollutants is less than the maximum discharge allowed by the environment:

$\sum_{t=1}^{2} \sum_{i=1}^{6} X_{i, t} \times S O_{i, t}+\sum_{t=1}^{2} \sum_{j=1}^{3} D_{j, t} \times S P_{j, t} \leq P L E_{1, t}$

$\sum_{t=1}^{2} \sum_{i=1}^{6} X_{i, t} \times N O_{i, t}+\sum_{t=1}^{2} \sum_{j=1}^{3} D_{i, t} \times N P_{j, t} \leq P L E_{2, t}$

where $S O_{i, t}$ and $N O_{i, t}$ represent the emission coefficients of sulfur dioxide and nitrogen oxides in the energy conversion process respectively. $N P_{j, t}$ and $S P_{j, t}$ represents the pollution emission coefficient of non-renewable resources (coal, diesel and gasoline) respectively (see Table 6). In each cycle, the emission of air pollutants is set to 100,000 tons.

\section{Results and Discussion}

This paper calculates the probability of $0.9,0.7,0.5,0.3$, and the four schemes obtained are shown in Table 7 . The schemes under different probabilities all meet the pollutant emission standards set in the constraint conditions. In the high probability scheme, the demand for each energy is very strict; therefore, the amount of coal imported from other places, the amount of local production, and the amount of coal-fired power generation are relatively higher. At this program, the credibility of the plan is high, with more air pollutants generated (Table 8). In the low probability scheme, the demand for each energy is relatively loose. Energy sources (coal and diesel) with more pollutants are imported and produced less (in the scenarios with probability 0.3 and 0.5 , the transfer of coal is reduced to 0 ), and the conversion amount of energy conversion methods that produce more pollutants is also less. In this case, the feasibility of the program is low, and the risk is high, but its pollution level and total system cost are lower.

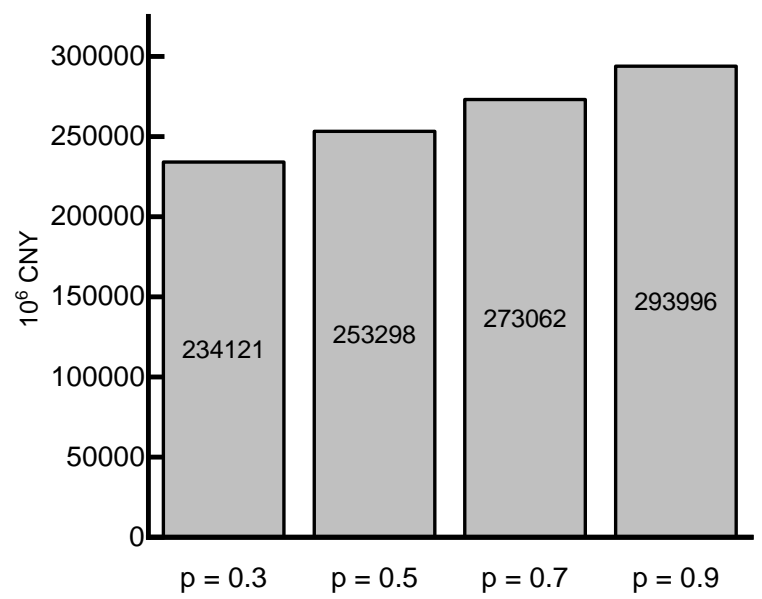

Figure 7. The minimum cost of the system obtained with different probabilities.

It can be seen from Figure 6 that as the probability decreases, the total system cost also decreases. This shows that the decision makers must take more risks when he or she wants to get a better planning scheme. The calculation results give the energy system structure optimization schemes under different satisfaction and risk levels.

The air pollutant emissions of the schemes under different probabilities meet the prescribed upper limit, the emission of air pollutants in the plan with low possibility is less. However, under this scheme, requirements for uncertain parameters in the system are relatively loose, the degree of risk increases accordingly.

In the construction and calculation process of the entire model, for the energy linear programming problem with resource and technical coefficients of normal type-2 fuzzy number with a completely normal secondary membership function, the possibility of the membership function is used to explain the inequality with fuzzy coefficients constraint. According to the definition of the possibility of membership function and its properties, the inequality constraints with fuzzy coefficients are transformed into concise equivalent forms. Finally, the decision-maker can obtain the optimal solution of the system planning for each different degree of possibility, and then choose the average according to the decision-maker's optimism. In the setting of the ideal general fuzzy coefficient in this article, the secondary membership function is set as an idealized normal 
Table 5. Conversion Factors of Different Power Generation Technologies

\begin{tabular}{lll}
\hline CFE $\left(10^{4} \mathrm{t} / \mathrm{PJ}\right)$ & $t=1$ & $t=2$ \\
\hline Coal-fired & $(0.56,0.67,0.06,0.05)$ & $(0.68,0.79,0.05,0.05)$ \\
Natural gas & $(0.5,0.6,0.05,0.05)$ & $(0.51,0.62,0.05,0.06)$ \\
Hydraulic & $(0.51,0.62,0.05,0.06)$ & $(0.47,0.6,0.04,0.06)$ \\
Wind & $(0.35,0.41,0.03,0.04)$ & $(0.39,0.46,0.04,0.05)$ \\
Nuclear & $(0.3,0.38,0.02,0.03)$ & $(0.36,0.38,0.03,0.04)$ \\
\hline
\end{tabular}

Table 6. Pollutant Emission Factor

\begin{tabular}{llllll}
\hline SP $(\mathrm{t} / \mathrm{PJ})$ & $t=1$ & $t=2$ & $\mathrm{NP}\left(10^{4} \mathrm{t} / \mathrm{PJ}\right)$ & $t=1$ & $t=2$ \\
\hline Coal & 0.9 & 0.8 & & 0.23 & 0.22 \\
Diesel & 0.25 & 0.22 & & 0.25 & 0.19 \\
Gasoline & 0.15 & 0.11 & $\mathrm{NO}\left(10^{4} \mathrm{t} / \mathrm{PJ}\right)$ & $t=1$ & 0.45 \\
\hline SO $\left(10^{4} \mathrm{t} / \mathrm{PJ}\right)$ & $t=1$ & $t=2$ & & $t=2$ \\
Coal-fired & 0.009 & 0.006 & 0.006 & 0.006 \\
Natural gas & 0.0002 & 0.00018 & 0.00015 & 0.00015 \\
\hline
\end{tabular}

Table 7. Calculation Results of Two Periods under Different Possibility $p$

\begin{tabular}{|c|c|c|c|c|}
\hline IA (PJ) & $p=0.9$ & $p=0.7$ & $p=0.5$ & $p=0.3$ \\
\hline Coal & $64.7,0$ & $3.6,0$ & 0,0 & 0,0 \\
\hline Diesel & $81,6.77$ & $69,63.1$ & $56.6,49.5$ & $44.4,36.1$ \\
\hline Gasoline & $57,9.60$ & $49.6,50.2$ & $41.6,40.5$ & $33.7,31$ \\
\hline Electric power & 0,0 & 0,0 & 0,0 & 0,0 \\
\hline Crude oil & $265.7,2875$ & $241.3,262.7$ & $217.5,238.5$ & $194.2,214.7$ \\
\hline Natural gas & $203.5,347$ & $197.6,335$ & $191.9,323.5$ & $186.4,312.5$ \\
\hline \multicolumn{5}{|l|}{ NA (PJ) } \\
\hline Coal & $227.1,0$ & $231.4,0$ & $179.8,0$ & $126.2,0$ \\
\hline Diesel & $60.7,70.8$ & $61.9,72.2$ & $63.3,73.8$ & $64.7,75.4$ \\
\hline Gasoline & $45.3,55.4$ & $45.9,56.1$ & $46.6,56.9$ & $47.3,57.7$ \\
\hline \multicolumn{5}{|l|}{$\mathrm{X}(\mathrm{PJ})$} \\
\hline Coal-fired & $341.6,708$ & $334.8,613.8$ & $328.1,530$ & $321.6,454.9$ \\
\hline Natural gas & $173.3,452.5$ & $169.8,443.4$ & $166.4,434.7$ & $163.1,426$ \\
\hline Hydraulic & $8.9,17.8$ & $8.7,17.4$ & $8.5,17.1$ & $8.3,16.7$ \\
\hline Wind & $53.5,99$ & $52.4,97$ & $51.3,95.1$ & $50.3,93.2$ \\
\hline Nuclear & $2.5,5.4$ & $2.4,5.3$ & $2.3,5.2$ & $2.3,5.1$ \\
\hline
\end{tabular}

Table 8. Pollutants from the Energy Conversion Process

\begin{tabular}{lllll}
\hline $\begin{array}{l}\text { Contaminant } \\
\text { type }\left(10^{4} \mathrm{t}\right)\end{array}$ & $p=0.9$ & $p=0.7$ & $p=0.5$ & $p=0.3$ \\
\hline $\mathrm{SO}_{2}$ & $3.10,4.32$ & $3.04,3.76$ & $2.98,3.25$ & $2.92,2.80$ \\
$\mathrm{NO}_{\mathrm{x}}$ & $2.07,4.31$ & $2.03,3.74$ & $1.99,3.24$ & $1.95,2.79$ \\
\hline
\end{tabular}

interval function, which makes the final result very ideal, and the ratio of the total cost to the corresponding probability is perfect gradient. The calculation result of the actual problem (using the fuzzy coefficient with the real secondary membership function data and further refine the gradient of the probability) will present a more realistic probability gradient map.

\section{Conclusions}

This paper improved the ranking method based on probability degree and extends the sorting of interval type-2 fuzzy numbers to the sorting of general fuzzy numbers. The new method is better at dealing with the uncertainty in linear pro- gramming problems, and the optimal solution of the objective function relaxation under different satisfaction can be obtained. By applying it in a simulated regional energy planning system, an optimization plan under different probabilities that meets the air pollutant emission indicators is obtained. In the application of regional energy system simulation planning, compared with the previous sorting algorithm, the new algorithm proposed in this paper takes the secondary membership function in the interval fuzzy number into consideration, the margin of error has been further reduced. However, when calculating the centroid of the secondary membership function of each point, depending on the number of discrete points, a larger calculation scale may be required when high accuracy is required (The secondary 
membership function in this article is set to a very ideal value). In the follow-up research, the calculation method of centroid trajectory can be improved to reduce the calculation scale and improve the performance of the model.

Acknowledgments. This research was supported by Youth Climbing Project (Grant No. XPDKT18010) of the Key Laboratory of Environmental biotechnology, Fujian Province University. The writers are very grateful to the editor and the anonymous reviewers for their insightful comments and suggestions.

\section{References}

Akram, M., Ullah, I., Allahviranloo, T. and Edalatpanah, S.A. (2021). Fully Pythagorean fuzzy linear programming problems with equality constraints. Computational and Applied Mathematics, 40(4), 1-30. https:// doi.org/10.1007/s40314-021-01503-9

Alizdeh, S. and Saeidi, S. (2020). Fuzzy project scheduling with critical path including risk and resource constraints using linear programming. International Journal of Advanced Intelligence Paradigms, 16(1), 4-17. https://doi.org/10.1504/IJAIP.2020.106687

BP in China. (2015). BP World Energy Statistics.http://www.bp.com/zh_ $\mathrm{cn} /$ china.html (accessed June 20, 2020).

Chen, L.P., Lin, X.M. and Xu, Y. (2019). Micro energy grid modeling and multi-objective optimal scheduling based on energy hub. Power System Protection and Control. 47(6), 9-16. (In Chinese)

Chen, Y. (2019). Study on sampling based discrete nie-tan algorithms for computing the centroids of general type-2 fuzzy sets. IEEE Access, 7, 156984-156992. https://doi.org/10.1109/ACCESS.2019.2949822

Chi, H.T.X. and Yu, V.F. (2018). Ranking generalized fuzzy numbers based on centroid and rank index. Applied Soft Computing Journal, 68, 283292. https://doi.org/10.1016/j.asoc.2018.03.050

Emeç, Ş. and Akkaya, G. (2021). Developing a new optimization energy model using fuzzy linear programming. Journal of Intelligent \& Fuzzy Systems, 1-14. https://doi.org/10.3233/JIFS-201994

Figueroa-Garcia, J.C., Chalco-Cano, Y. and Roman-Flores, H. (2018). Yager index and ranking for interval type-2 fuzzy numbers, 26(5), 27092718. IEEE Transactions on Fuzzy Systems. https://doi.org/10.1109/ TFUZZ. 2017.2788884

Guo, Z., Li, G., Zhou, M. and Feng, W. (2019). Two-stage robust optimal scheduling of regional integrated energy system considering network constraints and uncertainties in source and load, 43(9), 3090-2100. Dianwang Jishu/Power System Technology. https://doi.org/10.13335/j. 1000-3673.pst.2019.1113

Jin, L., Fu, H., Kim, Y., Wang, L., Li, Y. and Huang, G. (2019). A robust in- exact trapezoidal T2 fuzzy approach coupling possibility degrees for solid waste disposal allocation with integrated optimal greenhouse gas control under uncertainty. Journal of Cleaner Production, 221, 753-767. https://doi.org/10.1016/j.jclepro.2019.02.252

Kiptoo, M.K., Adewuyi, O.B., Lotfy, M.E., Senjyu, T., Mandal, P. and Abdel-Akher, M. (2019). Multi-objective optimal capacity planning for $100 \%$ renewable energy-based microgrid incorporating cost of demandside flexibility management. Applied Sciences (Switzerland), 9(18), 3855. https://doi.org/10.3390/app9183855
Li, G., Wang, R., Zhang, T. and Ming, M. (2018a). Multi-objective optimal design of renewable energy integrated CCHP system using PICEA-g. Energies, 11(4), 743. https://doi.org/10.3390/en11040743

Li, Y., Miao, S., Liu, J., Ye, C., Yin, B. and Yang, W. (2018b). Optimal allocation of energy storage system in PV micro grid considering uncertainty of demand response. Dianli Xitong Baohu Yu Kongzhi/Power System Protection and Control, 46(20), 69-77. https://doi.org/10.7667/P SPC171431

Liu, H., Liu, Q. and Zhang, L. (2019). Multi objective planning of cogeneration microgrid considering demand side cooperative response. Power System Protection and Control. 47(5), 43-51. (In Chinese)

Lu, Z.H., Li, Q. and Han, H.C. (2020). Model predictive control method of bi-level multi-objective fuzzy optimization for regional integrated energy system. Electric Power Construction. 41(12), 121-130. (In Chinese)

Mamdani, E.H. (1974). Application of fuzzy algorithms for control of simple dynamic plant. Proceedings of the Institution of Electrical Engineers, 121(12), 1585-1588. https://doi.org/10.1049/piee.1974.0328

Mendel, J.M. (2017). Type-2 fuzzy sets. In Uncertain rule-based fuzzy systems, 259-306. https://doi.org/10.1007/978-3-319-51370-66

Milanovic, M., Misita, M. and Komatina, N. (2020). Determination of the optimal production plan by using fuzzy AHP and fuzzy linear programming. Journal of Intelligent \& Fuzzy Systems, 38(4), 4315-4325. https:// doi.org/10.3233/JIFS-190913

Mohan, S., Kannusamy, A.P. and Sidhu, S.K. (2021). Solution of intuitionistic fuzzy linear programming problem by dual simplex algorithm and sensitivity analysis. Computational Intelligence, 37(2), 892-912. https://doi.org/10.1111/coin.12435

Naimi, M. and Tahayori, H. (2020). Centroid of polygonal fuzzy sets. Applied Soft Computing Journal, 95, 106519. https://doi.org/10.1016/j. asoc.2020.106519

National Bureau of Statistics of China. (2020). China statistical yearbook. Beijing: Chinese Statistical Bureau.

Srinivasan, A. and Geetharamani, G. (2016). Linear programming problem with interval type 2 fuzzy coefficients and an interpretation for its constraints. Journal of Applied Mathematics. https://doi.org/10.1155/ $2016 / 8496812$

Takagi, T. and Sugeno, M. (1985). Fuzzy Identification of Systems and Its Applications to Modeling and Control. IEEE Transactions on Systems, Man and Cybernetics, (1), 116-132. https://doi.org/10.1109/TSMC.19 85.6313399

Wu, D. and Mendel, J.M. (2009). A comparative study of ranking methods, similarity measures and uncertainty measures for interval type-2 fuzzy sets. Information Sciences, 179(8), 1169-1192. https://doi.org/10.1016/j. ins.2008.12.010

Wu, F.B., Shi, R.X. and Sang, B.Y. (2021). Optimization of integrated energy system considering energy cost and pollution emission. Thermal Power Generation. 50(2), 10-17. (In Chinese)

Zadeh, L.A. (1965). Fuzzy sets. Information and Control. https://doi.org/10. 1016/ S0019-9958(65)90241-X

Zeng, B., Hu, Q. and Zhang, Y. (2020). Probabilistic energy flow calculation and analysis of integrated electricity-gas systems with high penetration wind power. Taiyangneng Xuebao/Acta Energiae Solaris Sinica.

Zhao, T.Y. (2019). Modeling and Control of Type II Fuzzy System. Ph.D. Dissertation, School of Control Science and Engineering, Northwestern Polytechnical University, Xi'an, Shaanxi, China. (In Chinese) 